\title{
Paper and electronic text: psychophysiological features of reading and readers
}

\begin{abstract}
In article there are discussed psychophysiological parameters of reading the text at its perception by the subject on the paper and electronic medium of information. Results of researches of reading from printing and digital sources are systematized. As a result of the comparative analysis, it is established, that when reading the printing text, there are more optimum conditions for cognitive development of the reading subject. It is the basis for the recommendations of more correct use of electronic media of educational information in educational practice.
\end{abstract}

Keywords: reading, skill of reading, hypertext, internet search, brain activity, cognitive abilities
Volume 8 Issue 3 - 2017

Medvedskaya Al

Brest state University named after A.V. Pushkin, Belarus

Correspondence: Medvedskaya Al, Brest state University named after A.V. Pushkin, Mitskevicha, 28-207, Belarus, Email EMedvedskaja@mail.ru

Received: November 18, 2017 | Published: November 28, 2017

\section{Introduction}

Writing is one of key factors of formation of modern culture The cultural situation developing in the millennia with universal distribution of multimedia information devices, in which the written word acted as the main carrier of social experience, begins to change fundamentally. M. McLuhan, who has offered typology of civilizations by the leader like semiosis and who considered as the apologist of an electronic era, however, believed that the electric technology manages at our place, and we are mute, deaf, blind and insensible in the face of it collision with Guttenberg's technology. ${ }^{1}$ Among the current problems, which connected with transformation of a traditional printing information code, first of all, are noted growth of a functional dyslexia of younger generation ${ }^{2,3}$ and also increase the number of people who use a written language only under pressure of vital circumstances. ${ }^{3,4}$ One of determinants of these named problems is emergence of the increasing number of various media, which in competition the book begins to lose. However, it is thoughtthat the reason not only in escalating expansion of the multimodal, technically produced and amplifying information stream. Interaction with the paper text or the electronic device is set by absolutely different contexts of activity for the subject; therefore, it is quite naturally, as reading in different conditions will have the features. Carrying out the stated analysis per se is quite difficult, first of all, because of fast modification of sources of information and respectively, presence of quite small volume of empirical researches in this field. It is worth noting, there is a variety of the media today. Certain paper sources (like magazines) approach television by the figurativeness and minimal actually written text. As one of inveterate readers, who viewed such magazine, has precisely noted: I am not able to thumb through so quickly. At the same time, some electronic editions are in essence close to traditional, printing (for example, e-booksand hypertext). Because of the named restrictions of feature of perception of the text at the appeal of the subject to paper and electronic sources of a written language are considered quite technologically, i.e. without needs of the reader, so, and without contents. Though, certainly, the last is very important. As the leading tendency the Russian scientists noted a reading increasing reading pragmatization, prevalence of tool aspect (to be able) over informative (to know), present both at libraries, ${ }^{5-7}$ and on the Internet. ${ }^{8}$ However, despite the named reasons of difficulties, carrying out the comparative analysis of perception of the text from different data carriers is obviously necessary for definition of the directions of changes of reading in new sociocultural conditions and prevention of the possible negative psychological consequences connected with this transformation.

\section{Reading the text from paper}

The written language is formed on the basis of oral: originally the person learns to read, and then to write. Also, he reads during life more, then writes. Despite this reading, it is studied much less, than the letter, which connected with great difficulties of an operationalization of various stages of formation, which are frequently demanding special equipment. The American teacher and the media theorist $\mathrm{N}$. Postman considers that the leading forms of communication define the importance of culture, in other words, define the cognitive level of development of the person as its derivative. ${ }^{9} \mathrm{He}$ marks out the following lines of printing intellect: control of a state of the body (it is minimum condition for productive reading); ability to decipher actually word meaning behind an esthetics of letters, which forms quality of dispassionateness and objectivity at the reader ; ability to distinguish charm of words and the arguments built with their help; ability to remember questions, that in the process of reading to find answers (it is tolerance to the postponed answer); ability to formulation of counterarguments; a certain isolation, i.e. at understanding of readable to rely only on own intellectual opportunities. Thus, at the reader are formed abilities to concentration of attention and self-control, independence and criticality of thinking, dialogic of consciousness. It is worth noting, that the psychological qualities, that designated above correspond to model, which is described by the formula: reading technique + understanding $=$ functional literacy. The Russian scientists add to this model (but the truth, adjusted for the content of readable material) also sympathy and creation (integrative and associative concepts, feelings, meanings). ${ }^{10}$ Thus, can be added development of imagination, enrichment of experience and empathy to the functional characteristics, which named above. In general, it may be noted, that as the main result of development of the person reading is his ability to abstraction, ability to think without pictures acts.

\section{Reading the text from the computer screen}

As we know, an eye of skilled readers moves not linearly, but through fast leap from one point of fixing to another, ${ }^{11}$ i.e. process of reading also is carried out at these moments of fixings of eyes, which at 12-20times exceed time of their movement. There was no 
excluded regression, which reasons compliance of a context of its use standard, etc. In original experiments of T. Egorov it was proved, that restriction of a field of vision (in that case when only the read text was closed, and in that case, when there was no opportunity to see the forthcoming text) causes serious problems of reading. And problems are in its different components. One of them is deterioration in reading technique or transition from the visible word to pronouncing. Other problem belongs to disorganization of understanding. These data can serve as the reasonable assumption, reading from the computer screen, does not enhance skill of reading as it seldom is placed on the screen entirely, and an opportunity an eye to run forward pronouncing ${ }^{11}$ generally is minimum despite all possible options of technical reproduction of the page of the text. The additional proof of difficulties of reading from the computer screen the empirical calculations received by the Estonian psychologist Livshits $\mathrm{WM}^{12}$ can appear. Speed sensing of processing of information, which the scientist considers as an index of success of adaptation of the person to the environment, in case of achievement of a definite purpose appeared the purpose of its research. For assessment of this speed V. Livshits offered a formula, which has the quantity of the recognized letters and time spent for this process corresponds: $\mathrm{W}=\& \mathrm{I} / \& \mathrm{t}$ of bits (letter - hour), where \&I- an information increment, \& - adaptation time. During a series of experiments V. Livshits selected three factors of complexity of the environment decelerating information processing speed: form of presentation, character of a signal and invariant of the environment. In case of the verbal form of presentation of incentives at adult examines the speed of their recognition in comparison with visual was decelerated twice. More significantly speed is influenced by character of a signal, namely: in case of visual discrete (blinking) demonstration of letters speed was three times less, than in case of permanently the highlighted incentive. But the greatest impact on speed is exerted by invariance of incentives: regardless of the volume of the information absence in it certain steady reference points reduced the speed of its processing by 10times. There are just two last factors, when reading the majority of Web texts online: the discrete type of presentation of the text and inconstancy of the material. This allows saying, that the actual speed (if there is a task of processing of material) is not as high as it could be.

\section{Features of hypertexts}

The huge popularity of users was acquired by World Wibe Web-the part of the Internet based on the hypertext http (Hyper Text Transport Protocol) protocol. Unlike the idea of inclusion of T. Nelson assuming the active processing of the text by the reader, the idea of a hypertext link provides only his viewing. Analogy between the normal text link and a hyperlink (except for reduction of time of receiving last) is only apparent. The linearity of the printing text provides logical integrity of its contents, and thereby creates a natural opportunity for judgment by the reader. The traditional text is as the train, links and notes in it are like the pointers of the cities or landscape types flickering in a window approximately with the same probability to be visited, as well as other texts from which quotes and notes are taken..$^{13}$ The philosopher Nikitayev $\mathrm{BB}^{13}$ believes that the hypertext is the mechanical product constructed by means of mounting. As a result, visitors, having begun with one electronic page, will read different texts. Only at first glance everything depends on the purpose and the interests of different subjects of reading, really movement according to links reflects frames of the author of the source text and actually dictates its will. One of the most authoritative experts in the sphere of the Internet M. Castells considers it productive for the individual technology of release. ${ }^{14}$ At the same time, analyzing the existing practices of the appeal to network resources, it marks growth of network individualism: each personality creates the personal hypertext, which potentially can be a hindrance to search of common language, so and the general sense. As for creation of own text constructed on the Internet, the effect of the automatic letter is marked here. These are rather draft copies, something average between direct oral speech and specially created written text, reflection of pre-rational level of consciousness. Manifestation of the specified effect is more fragmentary (thesis) style of presentation without the detailed argumentation. ${ }^{15}$ Subtly noted, for a month of communication on the Internet at the forum devoted to imagination of the person thinly marked, he wrote 10 printed pages of the similar draft text while the speed of creation of traditional texts is much lower. The philologists researching different forms of speech activities on the Internet also come to the same conclusions. They note erosion boundaries between a spoken and written language, and appearance of new form of presentation of material as written informal conversation. ${ }^{16}$

\section{Specificity of internet search}

The American neurophysiologists Smoll G \& Vorgan $G,{ }^{17}$ generalizing results of researches of activity of a brain during an era of the Internet, believe that the brain of the modern person is in a condition of continuous digital stimulation. Scientists allocate two types of people: digital natives, i.e. representatives of younger generation, and digital emigrants, people of more advanced age, who need to master new technologies specially. The brain of digital native's functions in the mode of continuously scattered attention ${ }^{17}$ the attention watches everything at once, slides, without focusing on any object. The difference from a multitasking at which simultaneous performance by the subject of several tasks is subordinated to the accurate purposes consists in it. The brain of digital natives studies fast reactions and is exposed to permanent dopamine stimulation as which psychological consequence the habit of instant enjoying. At the same time, development of frontal lobes is slowed down, that worsens mental capacities and social skills. This deterioration is confirmed by results of other researches..$^{18}$ Attention should be drawn to results of one more experimental research conducted by Smoll G \& Vorgan G. ${ }^{17}$ By information search on the Internet, that is carried out it is computer competent users and illiterate (i.e. those who were never engaged in such activity, at the same time scientists stipulate how it was difficult to find him such volunteers), there are fundamental differences in activity of a brain showed by MRI. Namely, at the last the activity of dorsolateral frontal cortex, that controlling decision-making, synthesizing fragmentary information and the managing director of random access memory, hasn't been recorded. However, even more important, at these volunteers this zone of a brain began to become more active during the work with the Internet in only 5 days at 1 hour of interaction with the computer. And it is about people from 50 to $60 y e a r s$. Thus, these results allow drawing very important conclusion: it is computer illiterate very easily and can quickly become computerliterate, even at the level of functioning of a brain. There is no information that the same easy transition to other side is possible. But the information, which is available in a neuropsychology allow doubting it. According to A. Luriya's theory, the third block of a brain, which is morphological provided with frontal lobes of a brain, first of all prefrontal area of big hemispheres is responsible for any regulation of activity. Processes of regulation receive a long way of development in ontogenesis, and their formation is directly related with formation of the speech. At preschool children the regulating speech role still is weak. She submits to influence of direct impressions as operation of the visual analyzer is more closely connected with a word, than work 
of other sense organs. ${ }^{19}$ By 10years some components of arbitrary regulation, for example ability to check of hypotheses, generally rise to adult level. Others, in particular skills of planning, - aren't created also by 12 years..$^{20,21}$ In other words, those advantages in the speed of perception of information, which the brain gains thanks to intensive digital stimulation, at the same time, interfere with maturing of other its departments, which lag is hardly compensated at interaction with electronic devices. There is possible promotion more optimistic hypothesis, that by information search on the Internet at the user's strategies of naive training, which knowledge remains subconscious and doesn't verbalization. For modeling of naive education uses computer technologies and their effectiveness has proved, for example, in intensive training of doctors in diagnostic skills. ${ }^{22}$ However, in these cases education has purposeful and organized character: special training programs, which contents represent in a special way the picked-up material, that select experts in this sphere. Therefore, it is very doubtful; thatthere is similar training strategy of acquisition of knowledge at free and spontaneous use the various internet-resources.

\section{Conclusion}

The comparative analysis leads to the main conclusions. Reading is activities, which mastering leads to complication of the cognitive organization of the person. At the same time, enabling environment for such complication develop in case of perception of the paper texts, because reading from the computer screen doesn't promote formation of skill of reading in its operational or informative components. Therefore, it is optimally, that children learn to use electronic information resources, especially the Internet, only after automation of skill of reading. Use of digital technologies in education needs additional psychological researches actually from humanitarian line items, namely their value for development of pupils.

\section{Acknowledgements}

I commend to my 17-year-old son who's the real digital native. Thanks to its requests about classical paper textbooks on the most important subjects from his point of view, I had the idea of the presented analysis.

\section{Conflict of interest}

None.

\section{Funding}

None.

\section{References}

1. McLuhan M. Understanding media: external expansion of human Zhukovsky KANON-press, Kuchkovo pole, Moscow, Russia. 2003. p.464.

2. Zuckerman NA, Kovaleva GS, Kuznetsova MI. Well if you read Russian schoolchildren? Education. 2007;4:245.

3. Chudinova VP. The Functional illiteracy problem in developed countries Sociological studies. 1994;3:98-102.
4. Patzlaff R. Dead eyes: the physiological effects of television on children's development. Evidentis, Moscow, Russia. 2003. p.220.

5. Gudkov LD, Dubin BV. Russian libraries in the system of reproductive institutions: the context of prospects. Novoe literaturnoe obozrenie. 2005;74:166-202.

6. Dubin BV, Zorkaya NA. Reading in Russia-2008. Trends and challenges. Interregional center for library cooperation. Russia. 2008;80.

7. Stelmach VD. The modern Reader and literary community: the power of dialogue. Reading the world and the world of reading. Rudomino. Russia. 2003;145-156

8. Yakovleva E. Tell me what you read, it becomes clear what Russia will be like. Russia. 2015.

9. Postman N. TV creates a new type of person - adult child, Russia. 2015

10. Borodina VA. Psychology Techniques in the study of reading development. Bibliosphere. 2008;2:18-23.

11. Egorov TD. The Psychology of mastering reading skills. Russia. 2006;304.

12. Livshits WM. The Rate of human information processing and the factors of environmental complexity. The works on psychology. Tartu. 1976;4:139-146.

13. Nikitayev BB. The impact of the Internet on consciousness and knowledge structure. Institute of philosophy, Russian Academy of Sciences. Russia. 2004;73-93.

14. Castells M. The Internet Galaxy. Reflections on the Internet, business and society. (1st edn), Oxford University Press, Russia. 2003. p.304.

15. Katrechko SL. The Internet and consciousness: the concept of virtual person. The impact of the Internet on consciousness and knowledge structure. Institute of philosophy, Russian Academy of Sciences. Moscow, Russia. 2004. p.57-72.

16. Ilyina IA. Issues in the study and perception of hypertext in a multimedia environment: dis. kand. filol. Sciences. 2009;232.

17. Smoll G, Vorgan G. Brain online. People in the Internet age. Hummingbird. Moscow. Russia. 2011;352.

18. Nie NH, Hillygus DS. The impact of Internet use on Sociability. Timediary finding. IT \& Society. 2002;1:1-20.

19. Zaporozhets AV. The Mental development of the child. Pedagogy. Moscow, Russia. 1986;1:323.

20. Bezrucih MM. Central mechanisms of organization and regulation of voluntary movements in children 6-10 years. Message 1. Electrophysiological analysis of the process of preparation for the movements. Human physiology. 1997;23 (6):31-39.

21. Welch MC, Pennington BF, Grossier PB. Normal-developmental study of executive function. A window on prefrontal function in children. Developmental Neuropsychology. 1991;7(2):131-149.

22. Larichev OI, Naryzhny EV. Computer training procedural knowledge. Computers, brain, and cognition. Science. Russia. 2008. p.235-231. 